\title{
Assessment of Occlusal Contacts Leading to Orthodontic Relapse. A Case Study
}

\author{
Svitlana Koval* \\ Private practice limited to Orthodontics and TMD, SK Dental, Odessa, Ukraine
}

\begin{abstract}
Received: December 31, 2016; Accepted: January 11, 2017; Published: February 24, 2017
\end{abstract}
*Corresponding author: Dr.Svitlana Koval, MSc, Private practice limited to Orthodontics and TMD,SK Dental, Odessa, Ukraine, E-mail: sve.koval@icloud.com

\begin{abstract}
The probable mechanism of the orthodontic relapse was described in the article. T-scan III occlusal analysis system was used to examine occlusal contacts in post-orthodontic patient according to the author's protocol.
\end{abstract}

KeyWords: Occlusal contacts; Orthodontic relapse; T-scan III; Protrusion; Diastema; Disclusion time

\section{Introduction}

There is still a debate regarding the role of occlusal contacts in maintaining the stability of orthodontic treatment results. Occlusal contact distribution in post orthodontic patients was examined in various studies $[1,2,3]$. Authors put emphasis on the distribution of occlusal contacts in multi-bite recordings with the use of T-scan III occlusal analysis system [2], on the length of the Disclusion Time [DT] in orthodontically treated and nonorthodontically treated patients [1], the presence of non-working side contacts in later obtrusion and protrusion movements [3].

There is still a controversy regarding the role of canine guidance in protecting occlusion. The prevalence of canine guidance was stated to be less than group function in the examined group [4,5]. An effort was made to classify the stages of canine disclusion in another study [6]. The above mentioned study argues that already existing lateral movement pattern schemes are not enough to describe the full variety of occlusal patterns in lateral excursive movements. Studies $[4,5,6]$ consider both canine guidance and group function to be physiologic for the lateral excursive movements.

One of the earlier studies attempted to classify the lateral excursive movements with the use of T-scan II occlusal analysis system [7]. Classification consisting of 6 groups was described in this study. The groups were divided concerning the amount of disclusion from maximum interception into maximum canine excursion.

Another group of authors claim that gingival recessions and clefts are influenced by occlusal trauma [8], which has been studied in maximum interception and excursive movements.
The results of the study say that occlusion contributes to gingival recession and clefts.

Few studies accentuated on the point of teeth movement after the completion of orthodontic treatment and the mechanism that enables such movements.

Some authors used the T-scan III occlusal analysis after the completion of orthodontic treatment $[9,10]$.

The aim of this case report is to depict the probable existing mechanism of post-orthodontic tooth movement as a result of existing occlusal contacts.

\section{Case Description}

Twenty-three old patient, female, was treated orthodontic ally with non-removable bonded appliance on both jaws [figure.1] .After the completion of treatment non-removable bonded retainer was placed on the lingual surface the lower anterior teeth.

Regular check-up visits were planned: in one month after the completion of treatment, in 3 months, in 6 months, in 12 months after the completion of treatment.

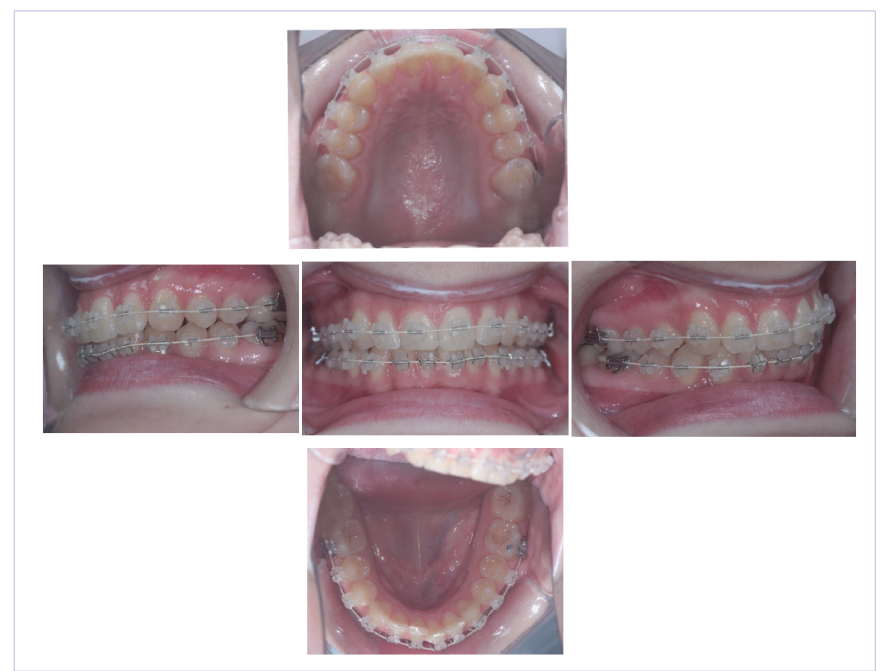

Figure 1 
During the first check-up visit the bonded retainer on the lower jaw was checked as well as the existence of occurring relapse in both jaws. The clinical examination revealed the occurrence of the small diastema between the teeth \#8 and 9 [figure. 2,3].

T-scan III occlusal analysis was performed to analyze the existing occlusal contacts. The author`s T-scan III protocol for the orthodontic patient examination consist of the following recordings: centric occlusion, multi-bite, left lateral excursion, right lateral excursion and protrusion recording.

The centric occlusion recording of the patient is shown in [figure. 4]. The diagram in the lower part shows the prolonged A-B period and the presence of the Z-form waves in the red and green force lines. The distribution of the teeth contacts is not perfect as well.

[Figure. 5] shows both the left and right lateral excursive movements at the start of the T-scan III occlusal analysis procedure. The most valuable information regarding these recordings is the duration of the Disclusion Time, which does not exceed 0,5 seconds in either recording.

The protrusive excursive movement was recorded. [Figure. 6] shows the summary of it. The diagram at the bottom of the picture shows the extended C-D period. The contact, persisting during the late opening is seen on the tooth \#2 with the force of 15,2 percents.

T-scan III computer guided occlusal adjustment [CGOA] was made to eliminate the persisting contacts during the protrusive movement. The main goal of the CGOA was to shorten the C-D time [Disclusion Time] in protrusive movement through eliminating the occlusal contacts that exist during the protrusion.

[Figure. 7] shows the result of the T-scan III CGOA procedure in the protrusive movement. The force-time diagram shows the C-D period being shortened and the time table shows the

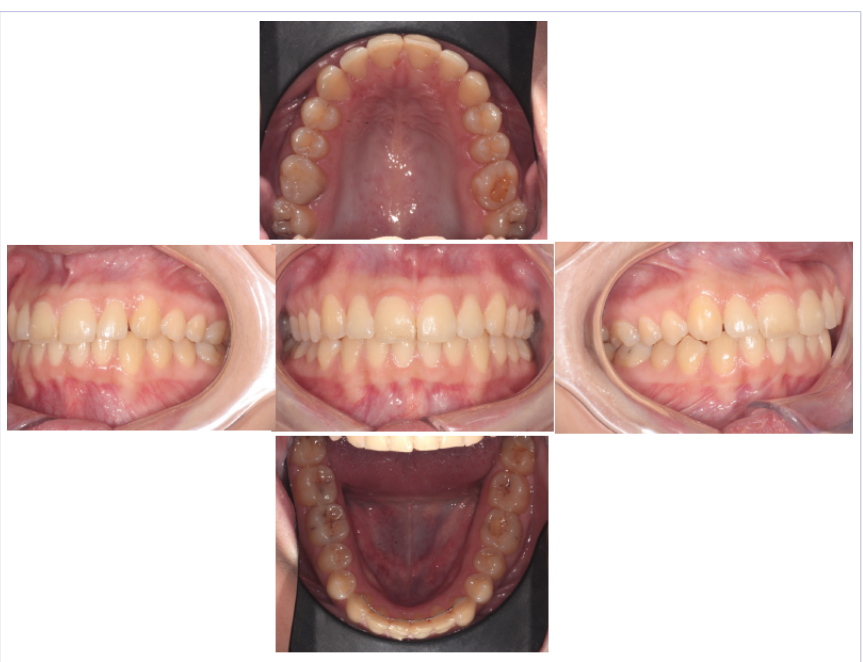

Figure 2

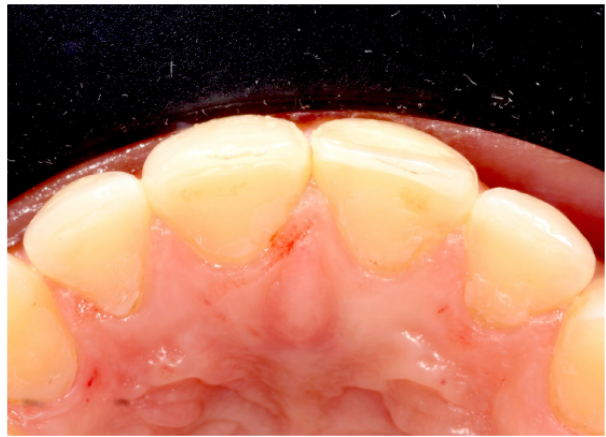

Figure 3

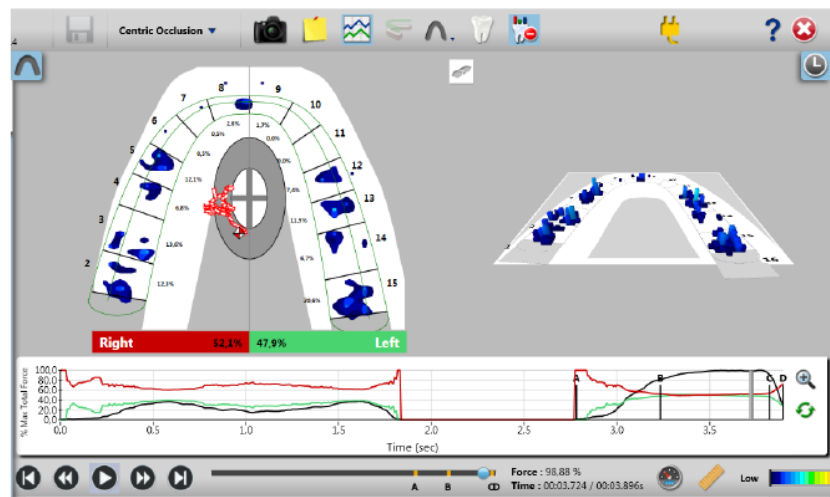

Figure 4: Centric occlusion at the start

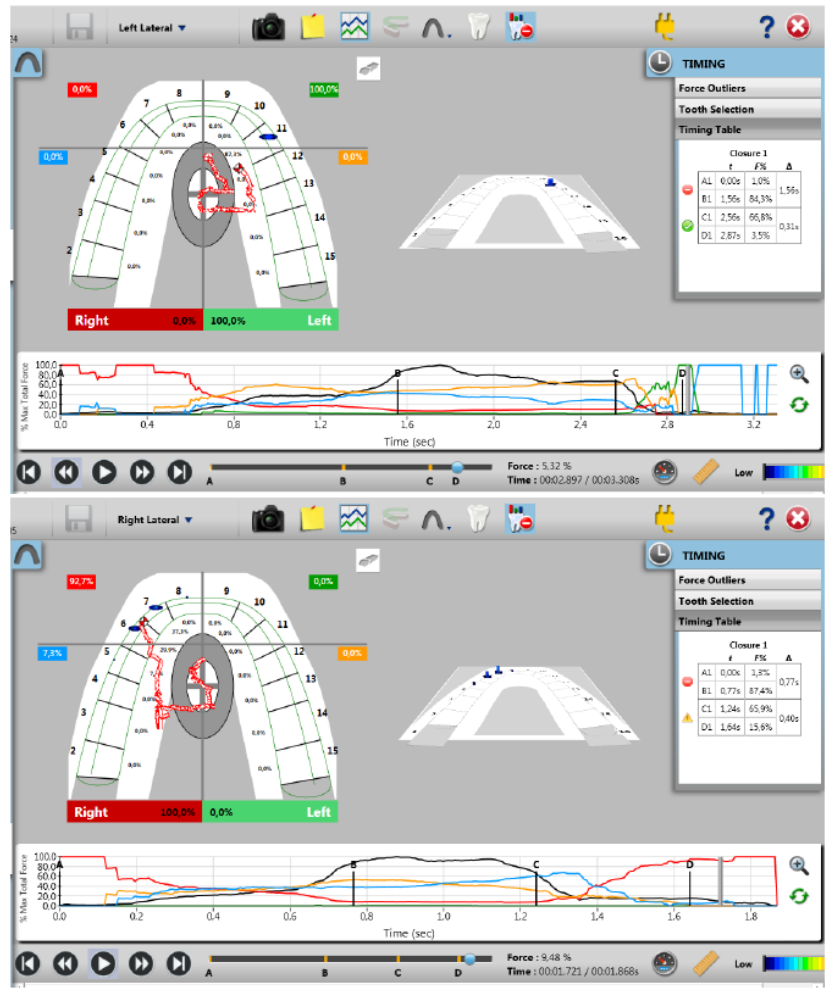

Figure 5: Left and Right lateral excursive movements 
Disclusion Time being 0,5 seconds. Although the contacts during the early protrusive movement are not totally eliminated, these contacts do not prolong the protrusion time for more than 0,5 seconds.

After the completion of the T-scan III CGOA procedure patient was left without any kind of orthodontic appliance on the upper jaw.

The next appointment was scheduled in 14 days. The diastema closed spontaneously. (Figure 8) shows the resulting diastema closure. The final result of diastema closure revealed stable during further appointments.

\section{Discussion}

According to our observations both diastema and spacing are caused by the buccal tooth inclination. The latter, according to our observations, is primarily caused by the excursive occlusal contacts.

The author's procedure of analyzing the existing occlusal contacts is described. T-scan III occlusal analysis is used to examine occlusal contacts during function. The standard protocol includes 5 recordings: centric occlusion, multi-bite, left and right excursive movement and protrusive movement.

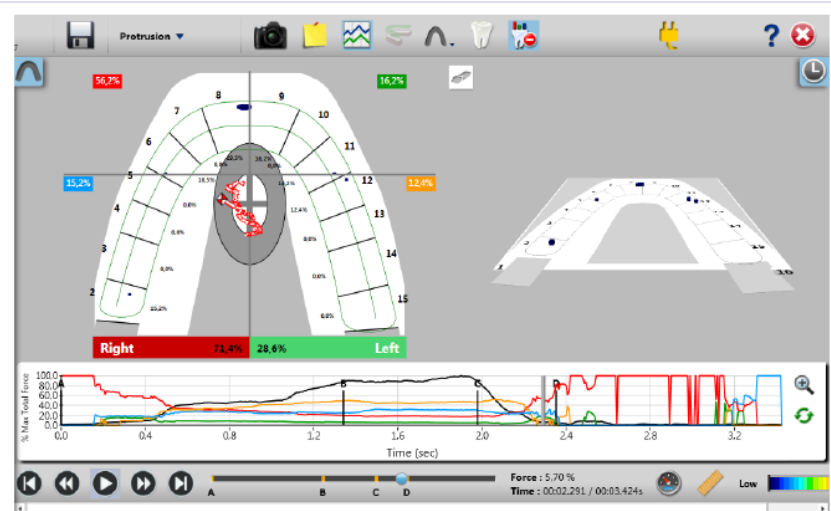

Figure 6: The protrusive excursion at the start of the T-scan III occlusal analysis procedure

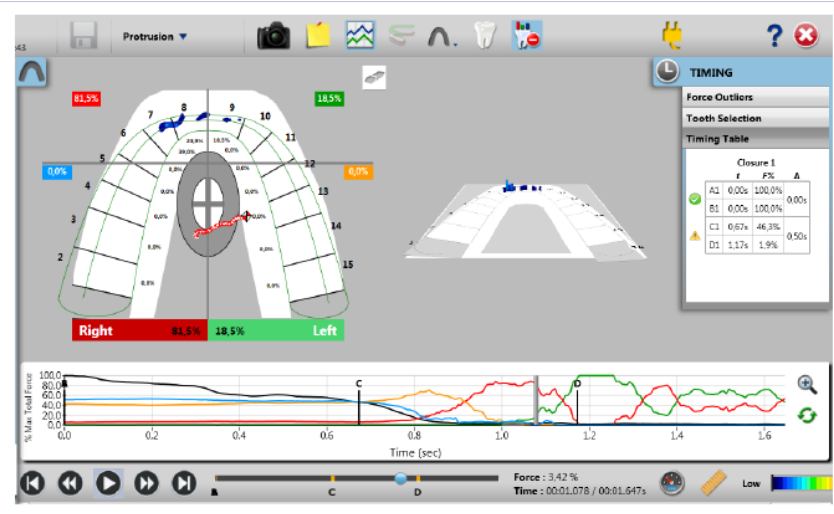

Figure 7: The result of the CGOA in protrusive movement

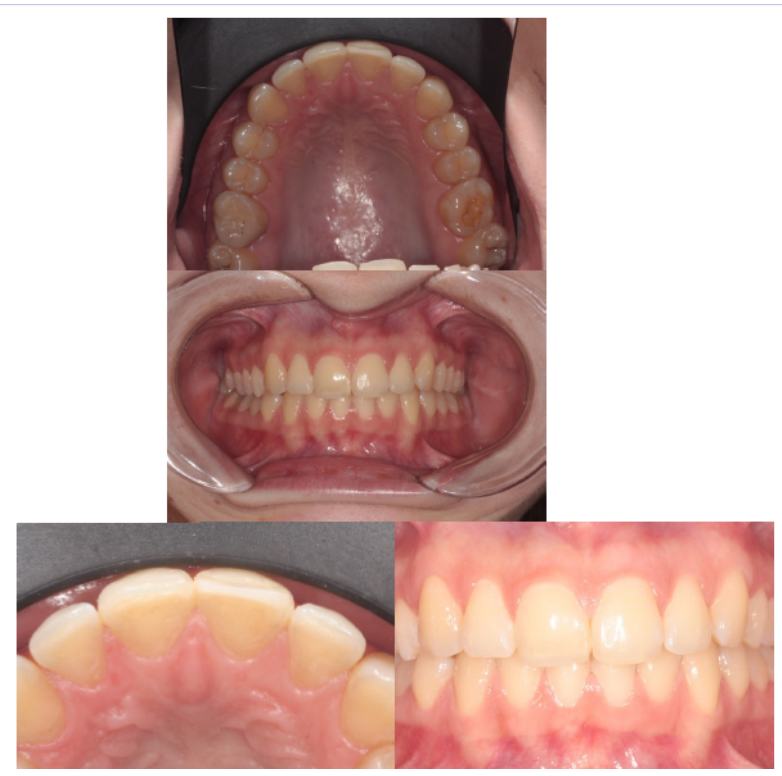

Figure 8

The centric occlusion recording is aimed to analyze the sequence of the appearing contacts, their duration, the duration of the A-B period, which indicates the time necessary for the teeth to occlude, the force distribution along the dental arches and the points of the greatest force located in each dental arch.

The multi-bite recording shows the repeatability of the A-B period duration, which is the first significant factor. It indicates the presence or absence of any potential contacts that might interfere with the closing movement of the jaws. The second value, that influences the occlusion, is the level of the overall force, exhibited during the closure cycle. In some cases the overall force, which is indicated by the black line in the diagram, can be lower in the second and third cycles of closure on the multi-bite recording.

Both left and right excursive movement is of extreme importance. The duration of the Disclusion Time [DT] is measured. The Disclusion Time is indicated by the C-D time span on the diagram. As stated in the literature, the duration of the C-D period should not exceed 0,5 seconds.

The protrusion movement is of the greatest interest in the described above case. The same rule is applied to the duration of the C-D period in protrusion movements as in lateral excursions. The C-D period duration is said to be not more than 0,5 seconds as well.

Our observations show that the length of the protrusion movement as well as the existence of the working contacts during the extended C-D period in protrusive movements are factors contributing to anterior spacing.

Elimination of the contacts that lengthen the C-D [e.g. The Disclusion Time] in protrusive movement removes the potential cause of the reoccurring spacing. Space opening mechanism 
implies, in our opinion that the buccal inclination of one of the anterior teeth is caused by the excessive pressure during the prolonged protrusive and lateral movements. The longer the excursive movement, the more likely the buccal inclination to occur. The localization of the excursive contact strongly influences the place of the space opening.

\section{Conclusion}

The probable mechanism for the occurring orthodontic relapse was described in this article. Further studies are necessary to prove the correlation between the buccal inclination of anterior teeth and the existing occlusal contacts.

\section{References}

1. Qadeer S, Abbas AA, Sarinnaphakorn L, Kerstein RB.Comparison of excursive occlusal force parameters in post-orthodontic and nonorthodontic subjects using T-Scan ${ }^{\circledR}$ III. Cranio. 2016;34(6):395-401. doi:10.1080/08869634.2015.1122277

2. Qadeer S, Abbas AA, Sarinnaphakorn L, Kerstein RB. Comparison of closure occlusal force parameters in post-orthodontic and nonorthodontic subjects using T-Scan ${ }^{\circledR}$ III DMD occlusal analysis.Cranio. 2016 Nov;34(6):395-401.

3. Milosevic A, Samuels RH. The post-orthodontic prevalence of temporomandibular disorder and functional occlusion contacts in surgical and non-surgical cases.J Oral Rehabil. 2000;27(2):142-149.
4. Francová K, Eber M, Zapletalová J. Functional occlusal patterns during lateral excursions in young adults.J Prosthet Dent. 2015113(6):571577. doi: 10.1016/j.prosdent.2014.12.004

5. Sapkota B, Gupta A. Pattern of occlusal contacts in lateral excursions (canine protection or group function). Kathmandu Univ Med J (KUMJ). 2014;12(45):43-47.

6. Singh A, Sangur R, Rao BL, Mahajan T. A clinical study to determine the pattern of occlusal contacts in lateral positions and its validity in classifying guidance patterns.J Indian Prosthodont Soc. 2013;13(2):101-107. doi: 10.1007/s13191-012-0210-1

7. Wang YL, Cheng J, Chen YM, Yip KH, Smales RJ, Yin XM. Patterns and forces of occlusal contacts during lateral excursions recorded by the T-Scan II system in young Chinese adults with normal occlusions.J Oral Rehabil. 2011;38(8):571-578. doi: 10.1111/j.13652842.2010.02194.x

8. Krishna Prasad D, Sridhar Shetty N, Solomon EG. The influence of occlusal trauma on gingival recession and gingival cleftsj Indian Prosthodont Soc. 2013;13(1):7-12. doi: 10.1007/s13191-012-0158-1

9. Koval S. T-scan occlusal analysis after adult orthodontic treatment. J Clinical Orthodontics. 2016;50(8):466-475.

10. Koval S. T-scan III Computed Guided Occlusal Adjustment in Orthodontic Relapse Patient.Procedure Description. EC Dental Science 6.2 (2016):1297-1308 